\title{
Haloperidol and clozapine antagonise amphetamine-induced disruption of latent inhibition of conditioned taste aversion
}

Journal Article

Author(s):

Russig, Holger; Kovacevic, Aneta; Murphy, Carol A.; Feldon, Joram

Publication date:

2003-11

Permanent link:

https://doi.org/10.3929/ethz-b-000052744

Rights / license:

In Copyright - Non-Commercial Use Permitted

Originally published in:

Psychopharmacology 170(3), https://doi.org/10.1007/s00213-003-1544-5 


\section{Holger Russig • Aneta Kovacevic • Carol A. Murphy • Joram Feldon \\ Haloperidol and clozapine antagonise amphetamine-induced disruption of latent inhibition of conditioned taste aversion}

Received: 5 November 2002 / Accepted: 17 May 2003 / Published online: 25 July 2003

(C) Springer-Verlag 2003

\begin{abstract}
Rationale: Latent inhibition (LI) describes a process by which repeated pre-exposure of a stimulus without any consequence retards the learning of subsequent conditioned associations with that stimulus. It is well established that LI is impaired in rats and in humans by injections of the indirect dopamine agonist amphetamine (AMPH), and that this disruption can be prevented by co-administration of either the typical neuroleptic haloperidol (HAL) or the atypical neuroleptic clozapine (CLZ). Objectives: Most of what is known of the pharmacology of LI is derived from studies using either the conditioned emotional response or the conditioned active avoidance paradigm. The goal of the present study was to determine whether these results would generalize to the conditioned taste aversion assay. Methods: We tested whether AMPH $(0.5 \mathrm{mg} / \mathrm{kg})$ pretreatment would disrupt LI of a conditioned aversion to sucrose, and if so, which stage of the procedure is critical for mediating the disruption; in addition, we tested whether HAL $(0.2 \mathrm{mg} /$ $\mathrm{kg})$ or CLZ $(5.0 \mathrm{mg} / \mathrm{kg})$ could restore such an expected LI disruption. Results: We determined that AMPH disrupted LI when it was injected before pre-exposure and prior to conditioning, but not if the rats were injected before either stage alone. When HAL or CLZ was given 40 min before AMPH (before both pre-exposure and conditioning), it blocked LI disruption. Conclusion: These results are in line with the pharmacology of LI as derived from other conditioning paradigms. We conclude that the pharmacological regulation of LI in the CTA paradigm is similar to what has been observed previously in the conditioned emotional response and the conditioned active avoidance paradigms.
\end{abstract}

H. Russig · A. Kovacevic · C. A. Murphy · J. Feldon ( Laboratory of Behavioral Neurobiology,

Swiss Federal Institute of Technology (ETH Zurich),

Schorenstrasse 16, 8603 Schwerzenbach, Switzerland

e-mail: feldon@behav.biol.ethz.ch

Tel.: +41-1-6557448

Fax: +41-1-6557203
Keywords Latent inhibition - Amphetamine .

Haloperidol · Clozapine $\cdot$ Conditioned taste aversion

\section{Introduction}

Latent inhibition (LI) is the phenomenon, occurring in a variety of species including humans and rats, whereby repeated unreinforced stimulus presentation retards subsequent conditioning to a stimulus (Lubow 1989). LI is believed by many investigators to be the product of learning to ignore irrelevant stimuli and, consequently, LI has been linked to the selectivity of attentional processing (Mackintosh 1975, Lubow et al. 1981, Lubow 1989). Disrupted LI in the rat is considered an animal model of cognitive/attentional deficits associated with schizophrenia, since it has been shown that acutely psychotic schizophrenic patients show both reduced LI and attentional deficits. However, LI is normalized during later episodes of this chronic disorder, possibly due to the effects of neuroleptic treatment (Baruch et al. 1988; Gray NS et al 1992a, 1995).

The pharmacology of LI in non-human subjects has been investigated using aversively-motivated LI procedures such as conditioned emotional response (CER), conditioned avoidance response (CAR), conditioned taste aversion (CTA), conditioned eyeblink, and conditioned freezing. LI can also be measured in appetitively motivated and discrimination learning procedures, but there has been only sporadic use of these methods (reviewed in Moser et al. 2000). In the CTA paradigm, a novel taste (CS, e.g. sucrose) is associated with illness induced by lithium chloride ( $\mathrm{LiCl}, \mathrm{US})$, thereby reducing subsequent sucrose preference during test. LI is demonstrated when animals pre-exposed (PE group) to the sucrose CS prior to CS-US pairing in the conditioning session show less sucrose aversion during the test session compared to nonpre-exposed (NPE group) animals. In comparison to CER and CAR procedures, only one CS-US pairing is necessary for strong conditioning and only a single pre- 
exposure to the CS induces robust LI in the CTA test (Russig et al., unpublished observation).

In animals and humans, the indirect dopamine agonist amphetamine (AMPH) disrupts LI; conversely, neuroleptic drugs with dopamine receptor antagonist activity, such as clozapine (CLZ) or haloperidol (HAL), can restore AMPH-induced LI disruption and enhance LI when given alone (Thornton et al. 1996; Kumari et al. 1999; Moser et al. 2000; Weiner 2000; Russig et al. 2002). A range of low doses of AMPH disrupt LI in CER (see Moser et al. 2000 for review) and CAR paradigms (Solomon et al. 1981; Weiner et al. 1988; Bakshi et al. 1995; De Oliveira Mora et al. 1999). Similarly, most of our knowledge about the capacity of compounds to reverse AMPH-induced disruption of LI is based on findings in the CER paradigm, with the exception of two studies which used CAR to show that chlorpromazine, CLZ and HAL are able to restore LI disruptions (Solomon et al. 1981, Russig et al. 2002). Ellenbroek et al. (1997) showed in rats that 0.25 and $0.5 \mathrm{mg} / \mathrm{kg}$ AMPH disrupted LI in a CTA paradigm. However, the ability of antipsychotic drugs such as HAL or CLZ to either restore disrupted LI or enhance normal LI has not yet been demonstrated in the CTA paradigm.

For both AMPH and neuroleptic effects on LI, the timing of drug administration is of critical importance in determining whether a drug effect is seen. In a typical experimental LI protocol in CER, the pre-exposure, conditioning and test sessions are carried out in separate sessions $24 \mathrm{~h}$ apart. When the experiment is conducted in this manner, AMPH must be administered before both pre-exposure and conditioning to disrupt LI (Weiner et al. 1984, 1988). However, a single administration of AMPH has been found sufficient to disrupt LI in humans and in rats if the pre-exposed CS duration was dramatically enhanced or the pre-exposure and conditioning phases were run in the same session (Gray NS et al. 1992b; Moran et al. 1996; Thornton et al. 1996; McAllister 1997). Based on these and other data, it has been argued that for AMPH to disrupt LI, it is actually the conditioning stage that is critical (Gray JA et al. 1995; Weiner and Feldon 1997). In the CTA paradigm, it was shown that AMPH disrupted LI when it was given before each of 3 pre-exposure days and the conditioning stage $24 \mathrm{~h}$ later (Ellenbroek et al. 1997). Thus, it remains an open question before which stage animals must be treated with AMPH in order to disrupt LI in the CTA paradigm. In contrast to the controversy over the relative importance of different experimental stages to AMPH-induced LI disruption, there is consistent evidence that the critical time for CLZ- or HAL-induced effects on LI is the conditioning stage. The effectiveness of these drugs in LI facilitation or reversal of AMPH-induced LI disruption was not altered by an additional injection before preexposure (Peters and Joseph 1993; Weiner 2000; Russig et al. 2002; Trimble et al. 2002).

For LI disruption in CER and CAR, the nucleus accumbens has been suggested to be the critical structure (Weiner and Feldon 1997; Murphy et al. 2000; Weiner
2000). However, recent experiments measuring c-Fos and employing intracerebral AMPH infusions have suggested that the striatum rather than the nucleus accumbens is the critical structure for LI disruption in CTA (Ellenbroek et al. 1997; Turgeon and Reichstein 2002). The same investigation methods used in a CER paradigm suggest a critical role of the nucleus accumbens rather than the striatum for disrupted LI (Solomon and Staton 1982; Sotty et al. 1996; Gray JA et al. 1997). With these differences in mind, very little is known about possible differences in the pharmacology of LI in CTA compared to other behavioral tests. An important step to address this issue is to investigate effects of AMPH, HAL and CLZ in this paradigm.

Therefore, we tested in the present study if the effects of AMPH, HAL and CLZ in CTA are comparable with the pharmacology of LI observed in other paradigms. We expected in experiment 1 that using a CTA procedure in which a 24-h delay took place between pre-exposure, conditioning and test sessions, LI would be disrupted if $0.5 \mathrm{mg} / \mathrm{kg}$ AMPH was administered $5 \mathrm{~min}$ before both the pre-exposure and conditioning sessions. We also anticipated that this effect of LI disruption should not occur if AMPH was administered either only before pre-exposure or only before conditioning. In addition, we expected that administration of $0.2 \mathrm{mg} / \mathrm{kg}$ HAL or $5.0 \mathrm{mg} / \mathrm{kg} \mathrm{CLZ} \mathrm{prior}$ to AMPH before both pre-exposure and conditioning would block the AMPH-induced LI disruption.

\section{Material and methods}

Animals

Male Wistar rats (Zur: WIST [HanIbm]; 250-350 g) obtained from our in-house specific-pathogen-free (SPF) breeding facility were used as subjects in these experiments. During the experiments, the animals were housed individually in Macrolon type III cages $(48 \times 27 \times 20 \mathrm{~cm})$ under a reversed light-dark cycle (lights on 1800 0600 hours $)$ in a temperature $\left(21 \pm 1^{\circ} \mathrm{C}\right)$ and humidity $(55 \pm 5 \%)$ controlled animal facility. Food (Kliba 3430, Klibamühlen, Kaiseraugst $\mathrm{CH}$ ) was available ad libitum in the home cages. All experiments were carried out between 8.00 a.m. and 1.00 p.m. during the dark phase of the light-dark cycle. All procedures were in agreement with Swiss Cantonal Veterinary Office regulations for animal experimentation.

\section{Drugs}

D-Amphetamine sulfate (Sigma Chemical Company, St Louis, USA) was dissolved in a $0.9 \% \mathrm{NaCl}$ solution to obtain the dosage of $0.5 \mathrm{mg} / \mathrm{kg}$ amphetamine (calculated as the salt). Vehicle-treated groups received $0.9 \% \mathrm{NaCl}$ solution. Haloperidol (HAL; JanssenCilag, Baar, Switzerland) was prepared from $5 \mathrm{mg}$ ampoules, in which the drug is present in $1 \mathrm{ml}$ of vehicle solution containing $6 \mathrm{mg}$ lactic acid. This solution was subsequently diluted with saline to obtain the required concentration of $0.2 \mathrm{mg} / \mathrm{kg}$ (final $\mathrm{pH}$ of 5.5). Clozapine (CLZ; Novartis, Switzerland) was first dissolved in $0.1 \mathrm{~N} \mathrm{HCL}$ in $0.9 \%$ saline solution and then neutralized to $\mathrm{pH} 5.5$ with $\mathrm{Na}_{2} \mathrm{CO}_{3}$ in a final concentration of $5.0 \mathrm{mg} / \mathrm{kg}$. Vehicle-treated animals were administered either HAL vehicle $(0.9 \%$ saline/lactic acid, pH 5.5) or CLZ vehicle (0.1 N HCL/0.9\% saline, pH 5.5). All solutions were freshly prepared and administered intraperitoneally in a volume of $1 \mathrm{ml} / \mathrm{kg}$. Lithium chloride $(\mathrm{LiCl}$, Sigma Chemical 
Company, St Louis, Mo. USA; $0.14 \mathrm{M}$ ) was dissolved in $0.9 \%$ $\mathrm{NaCl}$ and administered in a volume of $1.5 \%$ body weight.

\section{CTA apparatus}

Before each session animals were transferred from the home cage to a CTA test cage. The test cages were Macrolon cages $(42.5 \times 26.6 \times 15.0 \mathrm{~cm})$ designed in such a way that two drinking bottles could be attached and the spouts inserted through two holes in the anterior part of the cage. The water and sucrose intake of each animal were recorded by measuring the weight of the drinking bottles before and after each drinking session.

\section{CTA procedure}

Prior to the beginning of each experiment, animals were handled for $5 \mathrm{~min}$ each on 3 consecutive days and water deprived for the following 8 days. During the first 3 days of water deprivation, animals had access to water in the home cage for $1 \mathrm{~h}$ beginning at a time between 9.00 and 10.00 a.m. On the following 2 days, animals were exposed to the CTA test cages for $30 \mathrm{~min}$ where water was given. On the next day, the pre-exposure session with one drinking bottle was conducted in which animals were given either water (non-pre-exposed, NPE) or 5\% sucrose solution (pre-exposed, PE) for $30 \mathrm{~min}$ in the test cages. Up to this stage, the drinking bottles were switched once per exposure between the two holes in the test cages to avoid the development of preference for one hole over the other. On the next day, all animals experienced a conditioning session in which they were given access to $5 \%$ sucrose solution for 15 min immediately followed by an injection of lithium chloride ( $\mathrm{LiCl}, 0.14 \mathrm{M}, 1.5 \%$ of body weight) and were placed back in the home cage. During the next day, all animals were placed in the CTA cages and were given access to both $5 \%$ sucrose solution and water presented in two different bottles for $30 \mathrm{~min}$ at the same time (test session). Conditioned taste aversion was assessed by calculating the percent sucrose consumed ( $\mathrm{ml}$ sucrose consumed $\times 100 / \mathrm{ml}$ sucrose consumed $+\mathrm{ml}$ water consumed) on the test day. LI was assessed by comparing the degree of taste aversion between PE and NPE animals within each treatment group.

Experiment 1: effects of $0.5 \mathrm{mg} / \mathrm{kg}$ amphetamine injected before different experimental stages on the development of latent inhibition in a conditioned taste aversion paradigm

A dose of $0.5 \mathrm{mg} / \mathrm{kg}$ AMPH or saline was injected $5 \mathrm{~min}$ before the pre-exposure session, conditioning session, or before both sessions. A saline injection was given before all sessions without the AMPH treatment. The test session was conducted in a drug-free state. The dose of $0.5 \mathrm{mg} / \mathrm{kg}$ AMPH was selected because disrupted LI has been shown with this dose in the CER, passive avoidance and CTA paradigms (Killcross et al. 1994; Ellenbroek et al. 1997; De Oliveira Mora et al. 1999).

We excluded from analysis 12 animals that consumed less than $1.0 \mathrm{ml}$ of solution during the pre-exposure or conditioning session. The final number of animals in each of the eight conditions was: SAL/SAL NPE, $n=10$; SAL/SAL PE, $n=10$; AMPH/SAL NPE, $n=8$; AMPH/SAL PE, $n=9$; SAL/AMPH NPE, $n=8$; SAL/AMPH PE, $n=8 ;$ AMPH/AMPH NPE, $n=7 ;$ AMPH/AMPH PE, $n=8$

Experiment 2: effects of $0.2 \mathrm{mg} / \mathrm{kg}$ haloperidol on $0.5 \mathrm{mg} / \mathrm{kg}$ amphetamine-induced disruption of latent inhibition of conditioned taste aversion

Experiment 1 clearly showed that LI was reduced only if AMPH was given before both the pre-exposure and the conditioning sessions in the CTA paradigm. In experiment 2, either $0.2 \mathrm{mg} / \mathrm{kg}$ HAL or vehicle was injected $40 \mathrm{~min}$ prior to injection of either $0.5 \mathrm{mg} / \mathrm{kg}$ AMPH or saline, $5 \mathrm{~min}$ prior to the beginning of both the pre-exposure and conditioning sessions. During the test session all the animals were drug-free. The dose of $0.2 \mathrm{mg} / \mathrm{kg} \mathrm{HAL}$ was selected because dosages between $0.1 \mathrm{mg} / \mathrm{kg}$ and $0.5 \mathrm{mg} / \mathrm{kg}$ IP are effective in the reversal of AMPH $(1.0$ and $1.5 \mathrm{mg} / \mathrm{kg})$ induced disruption of LI (Warburton et al. 1994; Millan et al. 1998).

We excluded from the analyses five animals that consumed less then $1.0 \mathrm{ml}$ during the pre-exposure or the conditioning session. The final number of animals in each of the eight conditions was: SAL/SAL NPE, $n=10$; SAL/SAL PE, $n=10$; HAL/SAL NPE, $n=9$; HAL/SAL PE, $n=9$; SAL/AMPH NPE, $n=9$; SAL/AMPH PE, $n=9$; HAL/AMPH NPE, $n=10$; HAL/AMPH PE, $n=9$.

Experiment 3: effects of $5.0 \mathrm{mg} / \mathrm{kg}$ clozapine on $0.5 \mathrm{mg} / \mathrm{kg}$ amphetamine-induced disruption of latent inhibition of conditioned taste aversion

The experimental procedures were similar to those of experiment 2 , but instead of the typical antipsychotic drug HAL, the appropriate treatment groups received $5.0 \mathrm{mg} / \mathrm{kg}$ of the typical antipsychotic drug CLZ. The dosage of $5.0 \mathrm{mg} / \mathrm{kg}$ CLZ was selected because dosages between $2.0 \mathrm{mg} / \mathrm{kg}$ and $10.0 \mathrm{mg} / \mathrm{kg}$ IP are effective in the reversal of AMPH $(1.0$ and $1.5 \mathrm{mg} / \mathrm{kg})$ induced disruption of $\mathrm{LI}$ measured in a CER paradigm (Moran et al. 1996; Weiner et al. 1996; Millan et al. 1998).

We excluded from the analyses 14 animals that consumed less then $1.0 \mathrm{ml}$ during the pre-exposure or the conditioning session. The final number of animals in each of the eight conditions was: SAL/SAL NPE, $n=9$; SAL/SAL PE, $n=8$; CLZ/SAL NPE, $n=7$; CLZ/SAL PE, $n=9$; SAL/AMPH NPE, $n=8$; SAL/AMPH PE, $n=9$; CLZ/AMPH NPE, $n=8$; CLZ/AMPH PE, $n=8$.

\section{Statistics}

Statistical analysis of the data was conducted using StatView version 5.0.1. For all measurements in experiment 1 , we used a $2 \times 2 \times 2$ ANOVA design with the three between-subjects factors of drug treatment before pre-exposure (drug PE: SAL or AMPH), drug treatment before conditioning (drug COND: SAL or AMPH) and pre-exposure (PE or NPE). All measurements in experiments 2 and 3 were analyzed with $2 \times 2 \times 2$ ANOVA designs with three betweensubjects factors of drug treatment (SAL, AMPH), neuroleptic treatment (SAL, HAL or CLZ) and pre-exposure (NPE, PE). Whenever an interaction between two main factors was significant, the post-hoc Fisher's protected least significant difference test was applied.

\section{Results}

Experiment 1: effects of $0.5 \mathrm{mg} / \mathrm{kg}$ amphetamine injected before different experimental stages on the development of latent inhibition in a conditioned taste aversion paradigm

\section{Pre-exposure session}

Animals with access to sucrose (PE groups) consumed more solution than animals that had access only to water, as reflected by a main effect of pre-exposure $[F(1,60)=7.19, P<0.01$; see Fig. $1 \mathrm{~A}]$. The analysis also revealed a significant drug PExdrug COND $\times$ pre-exposure interaction $[F(1,60)=4.219, P<0.05]$. A post hoc analysis of this interaction revealed that the SAL/AMPH PE group drank more than the other groups, effects that were significant versus the SAL/AMPH NPE group 


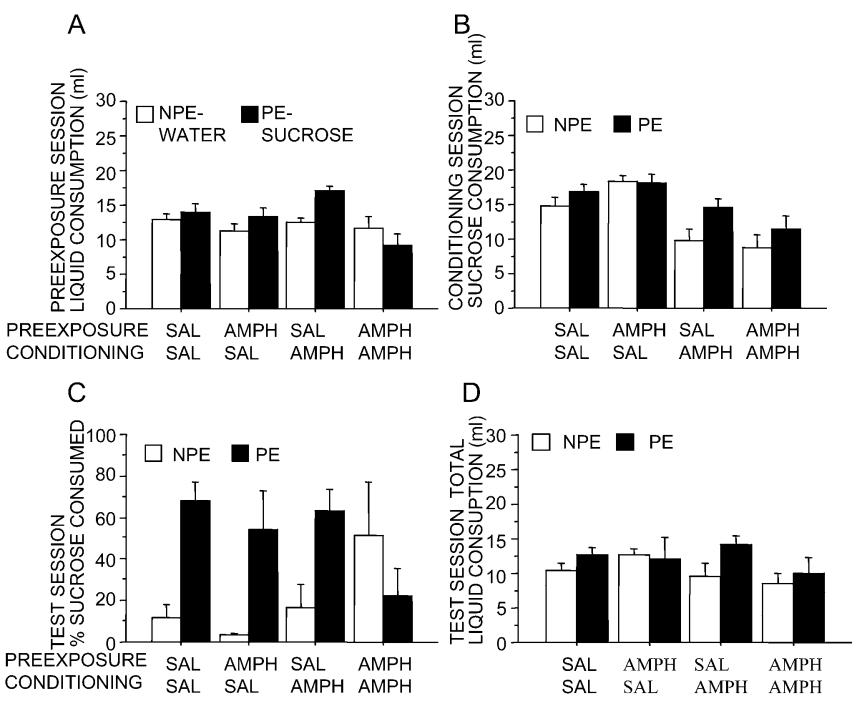

Fig. 1A-D Effects of amphetamine $(A M P H)$ or saline $(S A L)$ administration before different stages of an experiment measuring latent inhibition of conditioned taste aversion. A Liquid consumption (in $\mathrm{ml}$ ) during the pre-exposure session for sucrose preexposed $(P E)$ and non-pre-exposed (NPE) animals. NPE animals had access to water while PE animals received sucrose. B Sucrose consumption (in $\mathrm{ml}$ ) during the conditioning session. C Percent sucrose intake during the test session during which all animals had access to water and sucrose. D Total liquid consumption (in $\mathrm{ml}$, sucrose+water) during the test session. The groups were injected with saline $(S A L-S A L)$ or amphetamine $(A M P H-A M P H)$ before both the pre-exposure and the conditioning sessions, with amphetamine before pre-exposure and saline before conditioning (AMPH$S A L$ ) or with saline before pre-exposure and amphetamine before conditioning $(S A L-A M P H)$. Values are means \pm SEM

$(P=0.0001)$, AMPH/SAL NPE group $(P=0.0001)$ and AMPH/AMPH PE group ( $P=0.0003)$. No other significant main effects or interactions were found.

\section{Conditioning session}

Rats that had access to sucrose during the pre-exposure stage (PE groups) exhibited overall more sucrose intake compared to the NPE groups, as reflected by a significant main effect of pre-exposure $[F(1,60)=4.785, P<0.05$; Fig. 1B]. The analyses also revealed a significant main effect of drug during conditioning $[F(1,60)=29.916$, $P<0.0001]$, reflecting a reduced fluid intake for animals treated with AMPH before conditioning in comparison to SAL injected controls. This effect was more pronounced in animals that received AMPH also before pre-exposure, as reflected by a drug PExdrug COND interaction $[F(1,60)=4.387, P<0.05]$. There were no other significant main effects or interactions.

\section{Test session}

Overall expression of LI, as reflected by higher percent sucrose intake in PE compared with NPE animals, was confirmed by a significant main effect of pre-exposure $[F(1,60)=13.563, P<0.001$, Fig. $1 \mathrm{C}]$. However, the LI effect was not similar in all the treatment groups, as reflected by a significant drug PExpre-exposure interaction $[F(1,60)=5.681, P<0.03]$ and drug COND $\times$ preexposure interaction $[F(1,60)=7.161, P<0.01]$ and significant drug PExdrug CONDxpre-exposure interaction $[F(1,60)=4.314, P<0.05]$. Post hoc analyses revealed significantly more percent sucrose intake in PE compared to NPE animals (i.e. LI) in the groups that were treated with AMPH either only before pre-exposure (AMPH/ SAL, NPE versus $P E P=0.0046$ ), only before conditioning (SAL/AMPH, NPE versus PE $P=0.0094$ ) and in the control group (SAL/SAL, NPE versus PE, $P<0.0001$ ). No significant LI was obtained in animals treated with an AMPH injection both before pre-exposure and conditioning (AMPH/AMPH, group NPE versus PE, $P=0.2747)$. Comparing only the NPE groups of the different drug conditions AMPH/AMPH animals exhibited more percent sucrose intake than the AMPH/SAL NPE animals $(P=0.0259)$, but did not differ significantly $(P>0.353)$ from the other NPE groups. Within the PE groups, the AMPH/AMPH animals consumed significantly less percent sucrose compared to the SAL/AMPH $(P=0.0285)$ and the SAL/SAL $(P=0.0068)$ groups, but did not differ significantly from the AMPH/SAL group $(P=0.148)$. There was no significant main effect or interaction in the measure of total liquid consumption (water+sucrose, Fig. 1D).

Experiment 2: effects of $0.2 \mathrm{mg} / \mathrm{kg}$ haloperidol on $0.5 \mathrm{mg} /$ $\mathrm{kg}$ amphetamine-induced disruption of latent inhibition of conditioned taste aversion

\section{Pre-exposure session}

Animals with access to sucrose (PE groups) consumed more solution compared to the NPE groups that drank water, as reflected by a highly significant main effect of pre-exposure $[F(1,67)=17.936, P<0.0001$, Fig. 2A]. A significant drug treatment $\times$ pre-exposure interaction $[F(1,67)=4.903, P<0.05]$ reflected that this effect was more pronounced in the SAL-treated compared to the AMPH-treated animals.

\section{Conditioning session}

During the conditioning session, all groups exhibited similar sucrose intake and there were no significant main effects or interactions including the factors of preexposure, drug treatment or neuroleptic treatment (Fig. 2B). 

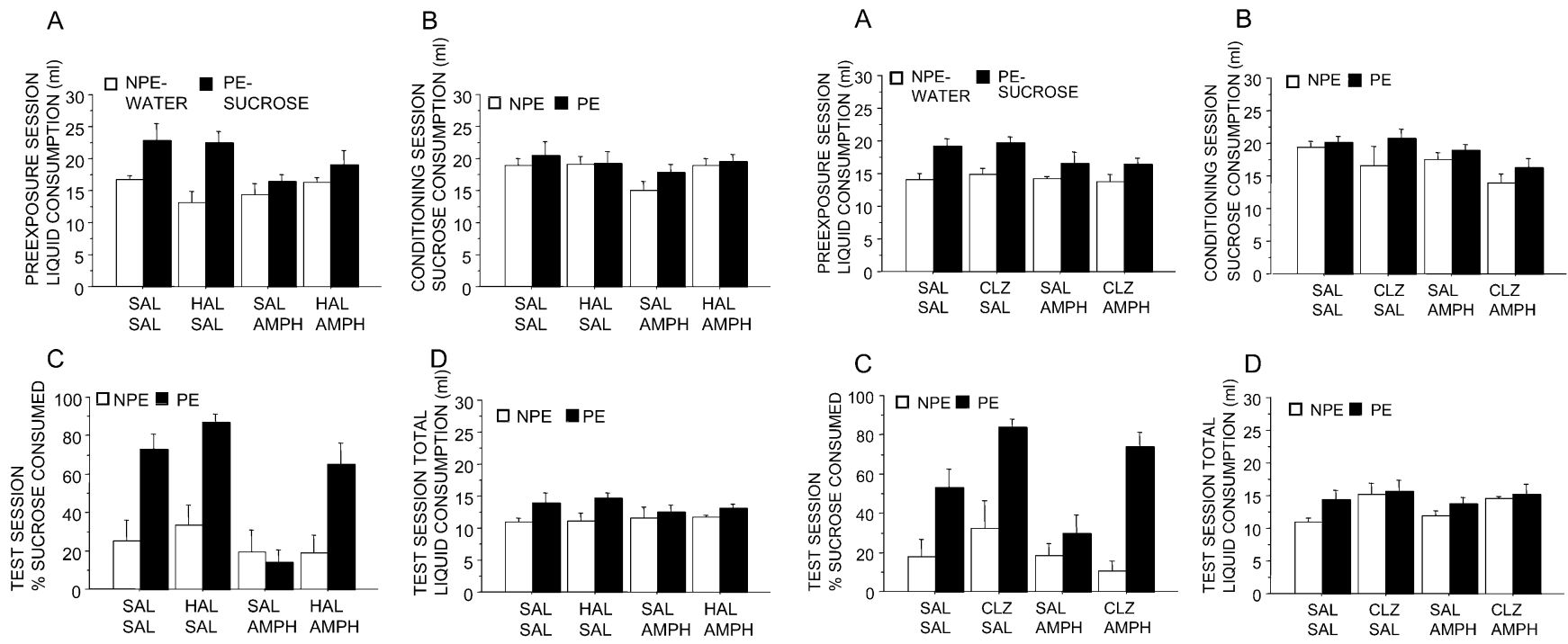

Fig. 2A-D Effects of amphetamine $(A M P H)$, haloperidol $(H A L)$ or saline $(S A L)$ administration before pre-exposure and conditioning sessions in an experiment measuring latent inhibition of conditioned taste aversion. A Liquid consumption (in $\mathrm{ml}$ ) during the preexposure session for sucrose pre-exposed $(P E)$ and non-preexposed $(N P E)$ animals. NPE animals had access to water while $\mathrm{PE}$ animals received sucrose. B Sucrose consumption (in $\mathrm{ml}$ ) during the conditioning session. C Percent sucrose intake during the test session during which all animals had access to water and sucrose. D Total liquid consumption (in $\mathrm{ml}$, sucrose+water) during the test session. Animals were injected with saline $(S A L-S A L)$, amphetamine $(S A L-A M P H)$, haloperidol $(H A L-S A L)$ or amphetamine and haloperidol $(H A L-A M P H)$ before both pre-exposure and test sessions. Values are means \pm SEM

\section{Test session}

The presence of LI, as indicated by higher percent sucrose intake in PE compared with NPE animals, was supported by a highly significant main effect of pre-exposure $[F(1,67)=29.982, \quad P<0.0001$; Fig. 2C]. AMPH-treated animals showed lower percent sucrose intake on the test day compared to the controls and this effect was mainly due to a reduction in the SAL/AMPH PE animals, as reflected by a significant main effect of drug treatment $[F(1,67)=15.208, P<0.0005]$ and a significant drug treatment $\times$ pre-exposure interaction $[F(1,67)=5.274, P<0.03]$. HAL treated rats showed enhanced percent sucrose intake compared to controls (main effect of neuroleptic treatment $[F(1,67)=7.762, P<0.01]$. Fishers post hoc comparisons between NPE and PE animals for all conditions revealed that LI was not present in AMPH treated animals and that the disruption was antagonized by pretreatment with HAL (NPE versus PE: SAL/SAL $P=0.0018$, HAL/ SAL $P=0.0002$, SAL/AMPH $P=0.7247$, HAL/AMPH $P=0.0048)$. A comparison of only the PE groups showed that the SAL/AMPH group showed reduced \% sucrose intake compared to the other three PE groups (all $P<0.0001)$ without a significant effect between these three groups. The NPE groups did not differ between the different treatment conditions in percent sucrose intake during the test session. In the analysis of overall

Fig. 3A-D Effects of amphetamine $(A M P H)$, clozapine $(C L Z)$ or saline $(S A L)$ administration before pre-exposure and conditioning sessions in an experiment measuring latent inhibition of conditioned taste aversion. A Liquid consumption (in $\mathrm{ml}$ ) during the preexposure session for sucrose pre-exposed $(P E)$ and non-preexposed (NPE) animals. NPE animals had access to water while $\mathrm{PE}$ animals received sucrose. B Sucrose consumption (in $\mathrm{ml}$ ) during the conditioning session. C Percent sucrose intake during the test session during which all animals had access to water and sucrose. D Total liquid consumption (in $\mathrm{ml}$, sucrose+water) during the test session. Animals were injected with saline $(S A L-S A L)$, amphetamine $(S A L-A M P H)$, clozapine $(C L Z-S A L)$ or amphetamine and clozapine $(C L Z-A M P H)$ before both pre-exposure and test sessions. Values are means \pm SEM

water+sucrose intake, PE animals drank more than NPE animals, as reflected by a significant main effect of preexposure $[F(1,67)=8.136, P<0.01]$, reflecting their increased sucrose intake (Fig. 2D). There were no significant interactions of total fluid intake with drug or neuroleptic treatment.

Experiment 3: effects of $5.0 \mathrm{mg} / \mathrm{kg}$ clozapine on $0.5 \mathrm{mg} / \mathrm{kg}$ amphetamine-induced disruption of latent inhibition of conditioned taste aversion

\section{Pre-exposure session}

Similarly to experiments 1 and 2, animals with access to sucrose (PE groups) consumed more solution compared to the NPE groups that drank water, as reflected by a highly significant main effect of pre-exposure $[F(1,58)=21.497$, $P<0.0001$, Fig. 3A]. In addition, AMPH pretreated animals consumed less solution during the 30 -min preexposure session compared to SAL pretreated animals irrespective of the neuroleptic pretreatment. This effect was reflected by a significant main effect of drug treatment $[F(1,58)=4.625, P<0.05]$. There were no additional significant main effects or interactions. 


\section{Conditioning session}

During the conditioning session, animals treated with AMPH consumed less sucrose solution compared to animals that received SAL $[F(1,58)=6.568, P<0.05$; Fig.3B]. A significant main effect of neuroleptic pretreatment $[F(1,58)=4.39, \quad P<0.05]$ reflected the fact that animals treated with CLZ showed reduced sucrose consumption during the conditioning session compared to vehicle treated animals. Rats that had access to sucrose during the pre-exposure stage (PE groups) exhibited overall more sucrose intake compared to the NPE groups, as reflected by a significant main effect of pre-exposure $[F(1,58)=4.733, \quad P<0.05]$. There were no significant interactions involving any of these three between-subjects factors.

\section{Test session}

The overall presence of LI was indicated by the fact that PE groups showed increased percent sucrose intake compared to NPE groups, as reflected by a highly significant main effect of pre-exposure $[F(1,58)=43.391$, $P<0.0001$; Fig. 3C]. Animals treated with AMPH before both the pre-exposure and the conditioning session showed reduced percent sucrose intake during the test session compared to the SAL-pretreated animals, as reflected by a significant main effect of drug treatment $[F(1,58)=4.972, P<0.05]$. On the other hand, animals previously treated with CLZ showed enhanced percent sucrose intake compared to animals treated with vehicle and this effect was more pronounced in the PE groups compared to the NPE groups. These effects were supported by a significant main effect of neuroleptic treatment $[F(1,58)=11.162, P<0.005]$ and a significant neuroleptic treatment $\times$ pre-exposure interaction $[F(1,58)=7.748, P<0.01]$. Fisher's post hoc comparisons between NPE and PE animals within all four conditions revealed that LI was not present in AMPH treated animals and that the disruption was antagonized by pretreatment with CLZ (NPE versus PE: SAL/SAL $P=0.0158$, CLZ/ SAL $P=0.0008, \quad$ SAL/AMPH $P=0.358, \quad$ CLZ/AMPH $P=0.0001)$. A post hoc comparison of only the PE groups for all conditions revealed a significantly reduced percent sucrose intake in the SAL/AMPH group compared to the other three groups (all $P<0.05$ ). In addition, CLZ/SAL PE animals exhibited enhanced percent sucrose intake compared to rats in the SAL/SAL PE condition $(P=0.0115)$. Similar Fisher's post hoc comparisons restricted to only the NPE groups of all four conditions did not reveal any significant outcome. The analysis of overall sucrose+water intake during the test session revealed a significant main effect of neuroleptic treatment $[F(1,58)=6.027$, $P<0.05]$, reflecting that animals previously treated with CLZ showed slightly enhanced solution intake compared to SAL treated animals (Fig. 3D). No other significant main effects or interactions were obtained in the analysis of overall solution intake during the test session.

\section{Discussion}

Experiment 1 showed that LI was disrupted in the CTA paradigm if an acute injection of $0.5 \mathrm{mg} / \mathrm{kg}$ AMPH was given before both the pre-exposure and the conditioning sessions. Animals that received AMPH either only before pre-exposure or only before conditioning exhibited normal LI. In experiments 2 and 3, administration of HAL or CLZ 40 min before the AMPH administration on both the pre-exposure and conditioning days blocked the AMPHinduced LI disruption.

During the pre-exposure phase in all three experiments, animals that had access to sucrose (PE groups) consumed more than animals that had only water to drink. These findings indicate that sucrose had more rewarding properties than water in general and was therefore more readily consumed, replicating the findings of Ellenbroek et al. (1997). During the conditioning session of experiments 1 and 3 but not 2, animals treated with AMPH before conditioning showed reduced sucrose intake compared to SAL-injected animals, thereby replicating findings of an AMPH-induced reduction of drinking (Foltin et al. 1983; Shepard 1988; Velazquez-Martinez et al. 1995). However, since the critical measure for LI is the difference between NPE and PE animals within a treatment group, and both were similarly influenced by AMPH we do not believe that these small and nonspecific effects confounded our results concerning LI in CTA.

In experiment 1 , we clearly showed that LI in CTA can be disrupted by AMPH only if the drug is administered before both pre-exposure and conditioning. A single injection given only before pre-exposure or conditioning left LI intact. These results are consistent with findings previously reported in the CER procedure (Weiner et al. 1984, 1988). Thus, in order to disrupt LI in CER or CTA protocols in which pre-exposure, conditioning and test sessions are separated by $24-\mathrm{h}$ intervals, it seems to be necessary to administer AMPH before both pre-exposure and conditioning. It has been suggested that processes of sensitization might be required for AMPH-induced LI disruption in experimental designs in which two injections must be given (Weiner et al 1988; Gray NS et al. 1995). We are currently examining whether, similar to CER, sensitization processes in CTA might also be responsible for AMPH-induced LI disruption.

The AMPH-induced LI disruption in all experiments was due to a significant reduction of percent sucrose intake in the AMPH/AMPH PE group compared to the control PE animals. However, in experiment 1 but not 2 or 3 , an additional non-significant increase in percent sucrose intake was observed in AMPH/AMPH NPE animals. It has been suggested that in order to obtain clearly interpretable results, drugs inducing LI disruption should selectively increase retarded CS-US conditioning in the treated PE group to levels expressed by the NPE group without altering conditioning in the NPE group itself (Lubow 1989; Weiner and Feldon 1997; Weiner 2000). It is unclear why in the first experiment the 
AMPH/AMPH NPE group showed somewhat increased percent sucrose intake; nevertheless, percent sucrose intake in the AMPH/AMPH NPE group did not differ significantly from that in the SAL/SAL NPE group. Given that elevated percent sucrose intake in the AMPH/AMPH NPE group was seen in only one of the three experiments, we believe that the effect was due to higher variability from random sampling error. It should be noted, moreover, that in both experiments, the animals belonging to the PE condition which was treated with AMPH before pre-exposure and conditioning showed less percent sucrose consumption compared with all other PE groups.

Based on the results obtained in experiment 1, we investigated the capacity of HAL and CLZ injected 45 min before pre-exposure and conditioning to block AMPH induced LI disruption in the CTA paradigm. To our knowledge, there are no published studies in the LI literature in which antipsychotic drugs and their influence on LI have been investigated in the CTA paradigm. In addition, there are only a few studies investigating antipsychotic compounds in LI procedures other than CER (Solomon et al. 1981, Weiner et al. 1987, Loskutova et al. 1990, Russig et al. 2002). Experiments 2 and 3 showed that HAL or CLZ injected before pre-exposure and conditioning blocked AMPH-induced LI disruption in a CTA paradigm. The disruption was clearly due to decreased percent sucrose intake in the SAL/AMPH PE group and HAL and CLZ antagonized this effect by increasing the percent sucrose intake of the HAL/AMPH and CLZ/AMPH PE groups to levels exhibited by the SAL/SAL PE groups. Interestingly, HAL/SAL animals exhibited slightly enhanced percent sucrose intake compared to the SAL/SAL group. This tendency was present in both the PE and the NPE groups but was most obvious in the PE groups. Nevertheless, the magnitude of LI was similar in the SAL/SAL and the HAL/SAL conditions, indicating that LI was not augmented by HAL in this experiment. In contrast, the results of experiment 3 suggest that CLZ induced enhancement of LI can be observed also in the CTA paradigm. Within experiment 3, CLZ/SAL PE animals exhibited significantly more percent sucrose intake compared to the SAL/SAL PE group and this effect of CLZ cannot be interpreted as general since the CLZ/SAL NPE animals did not significantly differ from the SAL/SAL NPE group. Enhanced LI by HAL might also be detectable in future experiments using different dosages, or if the procedural parameters were manipulated in such a way that LI in controls was less pronounced, given indications that neuroleptic-induced LI enhancement is best seen on a background of no LI in the controls (Weiner and Feldon 1997; Moser et al. 2000). LI in the controls could be reduced by shortening the duration of the pre-exposure session, restricting sucrose intake during pre-exposure, or reducing the dose of $\mathrm{LiCL}$ and thereby reducing the strength of the CS-US association (Weiner 2000). Finally, the LI effects of experiments 2 and 3 cannot be due simply to an effect of the various treatments on drinking behavior per se, because drinking was not influenced by either the AMPH or the
HAL treatment during the test session. Moreover, although CLZ enhanced the total solution intake during the test session, the analysis did not reveal any statistically significant interactions with the factors of drug treatment or pre-exposure, suggesting that this effect cannot be responsible for the observed effects on LI in the different drug conditions.

Theoretically, there are some potential problems of interpretation in using CTA as a paradigm to investigate LI. AMPH effects on LI in CTA might be confounded by the fact that AMPH itself can induce conditioned taste aversion (Miller and Miller 1983; Greenshaw and Dourish 1984; Goudie and Newton 1985). In our experiments 1 and 2, there were no significant differences in sucrose intake between the pre-exposure and the conditioning sessions in animals that received AMPH before sucrose pre-exposure (AMPH PE group). Consequently, it is very unlikely that our low dose of AMPH induced an independently conditioned taste aversion in these rats. Another criticism regarding measurements of LI in CTA is that pre-exposure to the sucrose CS in these experiments is not really without a consequence because the CS reduces thirst in water-deprived animals and provides a rewarding taste. Therefore, it could be argued that PE animals do not really learn to ignore the CS; in fact, the opposite is the case-they learn to appreciate the sweet solution, a fact that perhaps strengthens the LI effect (Moser et al. 2000). Nevertheless, LI was disrupted by a very low dose of AMPH in the present experiment. It is interesting that the pharmacology of AMPH, HAL and CLZ in the LI CTA paradigm is very similar to results obtained in paradigms not confounded by these theoretical criticisms, such as the CAR and CER procedures.

Taken together, our results show that 1) LI is disrupted in the CTA paradigm if AMPH is administered before both pre-exposure and conditioning and 2) this disruption can be blocked by HAL and CLZ. These results indicate that the pharmacology of LI regulation by dopamine agonists and antagonists is similar in the CTA paradigm to that of other tests such as CAR and CER.

Acknowledgements This study was supported by the Swiss Federal Institute of Technology (ETH-Zurich, Switzerland). We would like to thank the staff of the animal facility for their care and maintenance of the animals used in this study, Mr. Peter Schmid for his valuable technical assistance and Mrs. Jane Fotheringham for her editorial help.

\section{References}

Bakshi VP, Geyer MA, Taaid N, Swerdlow NR (1995) A comparison of the effects of amphetamine, strychnine and caffeine on prepulse inhibition and latent inhibition. Behav Pharmacol 6:801-809

Baruch I, Hemsley DR, Gray JA (1988) Differential performance of acute and chronic schizophrenics in a latent inhibition task. J Nerv Ment Dis 176:598-606

De Oliveira Mora P, Fouquet N, Oberling P, Gobaille S, Graeff FG, Sandner G (1999) A neurotoxic lesion of serotonergic neurones using 5,7-dihydroxytryptamine does not disrupt latent inhibi- 
tion in paradigms sensitive to low doses of amphetamine. Behav Brain Res 100:167-175

Ellenbroek BA, Knobbout DA, Cools AR (1997) The role of mesolimbic and nigrostriatal dopamine in latent inhibition as measured with the conditioned taste aversion paradigm. Psychopharmacology 129:112-120

Foltin RW, Woolverton WL, Schuster CR (1983) Effects of psychomotor stimulants, alone and in pairs, on milk drinking in the rat after intraperitoneal and intragastric administration. J Pharmacol Exp Ther 226:411-418

Goudie AJ, Newton T (1985) The puzzle of drug-induced conditioned taste aversion: comparative studies with cathinone and amphetamine. Psychopharmacology 87:328-333

Gray JA, Joseph MH, Hemsley DR, Young AM, Warburton EC, Boulenguez P, Grigoryan GA, Peters SL, Rawlins JN, Taib CT et al. (1995) The role of mesolimbic dopaminergic and retrohippocampal afferents to the nucleus accumbens in latent inhibition: implications for schizophrenia. Behav Brain Res 71:19-31

Gray JA, Moran PM, Grigoryan G, Peters SL, Young AM, Joseph MH (1997) Latent inhibition: the nucleus accumbens connection revisited. Behav Brain Res 88:27-34

Gray NS, Hemsley DR, Gray JA (1992a) Abolition of latent inhibition in acute, but not chronic, schizophrenics. Neurol Psychiatr Brain Res 1:83-89

Gray NS, Pickering AD, Hemsley DR, Dawling S, Gray JA (1992b) Abolition of latent inhibition by a single $5 \mathrm{mg}$ dose of $d$ amphetamine in man. Psychopharmacology 107:425-430

Gray NS, Pilowsky LS, Gray JA, Kerwin RW (1995) Latent inhibition in drug naive schizophrenics: relationship to duration of illness and dopamine $\mathrm{D}_{2}$ binding using SPET. Schizophr Res 17:95-107

Greenshaw AJ, Dourish CT (1984) Differential aversive stimulus properties of beta-phenylethylamine and of $d$-amphetamine. Psychopharmacology 82:189-193

Killcross AS, Dickinson A, Robbins TW (1994) Amphetamineinduced disruptions of latent inhibition are reinforcer mediated: implications for animal models of schizophrenic attentional dysfunction. Psychopharmacology 115:185-195

Kumari V, Cotter PA, Mulligan OF, Checkley SA, Gray NS, Hemsley DR, Thornton JC, Corr PJ, Toone BK, Gray JA (1999) Effects of $d$-amphetamine and haloperidol on latent inhibition in healthy male volunteers. J Psychopharmacol 13:398-405

Loskutova LV, Luk'yanenko FY, Il'yuchenok RY (1990) Interaction of serotonin- and dopaminergic systems of the brain in mechanisms of latent inhibition in rats. Neurosci Behav Physiol 20:500-505

Lubow RE (1989) Latent inhibition and conditioned attention theory. Cambridge University Press, Cambridge, UK

Lubow RE, Weiner I, Schnur P (1981) Conditioned attention theory. In: Bower GH (ed) The psychology of learning and motivation. Academic Press, New York

Mackintosh NJ (1975) A theory of attention: variations in the associability of stimuli with reinforcement. Psychol Rev 82:276-298

McAllister KH (1997) A single administration of d-amphetamine prior to stimulus pre-exposure and conditioning attenuates latent inhibition. Psychopharmacology 130:79-84

Millan MJ, Schreiber R, Dekeyne A, Rivet JM, Bervoets K, Mavridis M, Sebban C, Maurel-Remy S, Newman-Tancredi A, Spedding M, Muller O, Lavielle G, Brocco M (1998) S 16924 ((R)-2-[1-[2-(2,3-dihydro-benzo[1,4] dioxin-5-yloxy)-ethyl]pyrrolidin-3yl]-1-(4-fluoro-phenyl)-ethanone), a novel, potential antipsychotic with marked serotonin (5-HT)1A agonist properties: II. Functional profile in comparison to clozapine and haloperidol. J Pharmacol Exp Ther 286:1356-1373

Miller DB, Miller LL (1983) Bupropion, $d$-amphetamine, and amitriptyline-induced conditioned taste aversion in rats: dose effects. Pharmacol Biochem Behav 18:737-740
Moran PM, Fischer TR, Hitchcock JM, Moser PC (1996) Effects of clozapine on latent inhibition in the rat. Behav Pharmacol 7:42_ 48

Moser PC, Hitchcock JM, Lister S, Moran PM (2000) The pharmacology of latent inhibition as an animal model of schizophrenia. Brain Res Rev 33:275-307

Murphy CA, Pezze M, Feldon J, Heidbreder C (2000) Differential involvement of dopamine in the shell and core of the nucleus accumbens in the expression of latent inhibition to an aversively conditioned stimulus. Neuroscience 97:469-477

Peters SL, Joseph MH (1993) Haloperidol potentiation of latent inhibition in rats: evidence for a critical role at conditioning rather than pre-exposure. Behav Pharmacol 4:183-186

Russig H, Murphy CA, Feldon J (2002) Clozapine and haloperidol reinstate latent inhibition following its disruption during amphetamine withdrawal. Neuropsychopharmacology 26:765777

Shephard RA (1988) Valproate enhances fluid consumption suppressed by shock or neophobia, but not by partial satiation or $d$-amphetamine, in rats. Life Sci 43:897-904

Solomon PR, Staton DM (1982) Differential effects of microinjections of $d$-amphetamine into the nucleus accumbens or the caudate putamen on the rat's ability to ignore an irrelevant stimulus. Biol Psychiatry 17:743-756

Solomon PR, Crider A, Winkelman JW, Turi A, Kamer RM, Kaplan LJ (1981) Disrupted latent inhibition in the rat with chronic amphetamine or haloperidol-induced supersensitivity: relationship to schizophrenic attention disorder. Biol Psychiatry 16:519-537

Sotty F, Sandner G, Gosselin O (1996) Latent inhibition in conditioned emotional response: c-fos immunolabelling evidence for brain areas involved in the rat. Brain Res 737:243254

Thornton JC, Dawe S, Lee C, Capstick C, Corr PJ, Cotter P, Frangou S, Gray NS, Russell MA, Gray JA (1996) Effects of nicotine and amphetamine on latent inhibition in human subjects. Psychopharmacology 127:164-173

Trimble K, Bell R, King D (2002) Effects of the selective dopamine D(1) antagonists NNC 01-0112 and SCH 39166 on latent inhibition in the rat. Physiol Behav 77:115

Turgeon SM, Reichstein DA (2002) Decreased striatal c-Fos accompanies latent inhibition in a conditioned taste aversion paradigm. Brain Res 924:120-123

Velazquez Martinez DN, Valencia Flores M, Lopez Cabrera M, Villarreal JE (1995) Effects of indorenate on food intake: a comparison with fenfluramine and amphetamine. Psychopharmacology 117:91-101

Warburton EC, Joseph MH, Feldon J, Weiner I, Gray JA (1994) Antagonism of amphetamine-induced disruption of latent inhibition in rats by haloperidol and ondansetron: implications for a possible antipsychotic action of ondansetron. Psychopharmacology 114:657-664

Weiner I (2000) The latent inhibition model of schizophrenia. In: Myslobodsky MS, Weiner I (eds) Contemporary issues in modeling psychopathology. Kluwer, Boston

Weiner I, Feldon J (1997) The switching model of latent inhibition: an update of neural substrates. Behav Brain Res 88:11-25

Weiner I, Lubow RE, Feldon J (1984) Abolition of the expression but not the acquisition of latent inhibition by chronic amphetamine in rats. Psychopharmacology 83:194-199

Weiner I, Feldon J, Katz Y (1987) Facilitation of the expression but not the acquisition of latent inhibition by haloperidol in rats. Pharmacol Biochem Behav 26:241-246

Weiner I, Lubow RE, Feldon J (1988) Disruption of latent inhibition by acute administration of low doses of amphetamine. Pharmacol Biochem Behav 30:871-878

Weiner I, Shadach E, Tarrasch R, Kidron R, Feldon J (1996) The latent inhibition model of schizophrenia: further validation using the atypical neuroleptic, clozapine. Biol Psychiatry 40:834-843 